\title{
Advance Care Planning Improves Psychological Symptoms But Not Quality of Life and Preferred End-of-Life Care of Patients With Cancer
}

\author{
Siew Tzuh Tang, DNSc ${ }^{\mathrm{a}, \mathrm{b}, \mathrm{c}}$; Jen-Shi Chen, MD d,e; Fur-Hsing Wen, PhDf; Wen-Chi Chou, MD d,e; \\ John Wen-Cheng Chang, MDd,e; Chia-Hsun Hsieh, MDd,e; and Chen Hsiu Chen, PhDg
}

\begin{abstract}
Background: This study was conducted to examine whether a longitudinal advance care planning (ACP) intervention facilitates concordance between the preferred and received life-sustaining treatments (LSTs) of terminally ill patients with cancer and improves quality of life ( $\mathrm{Q} \circ \mathrm{L})$, anxiety symptoms, and depressive symptoms during the dying process. Patients and Methods: Of 795 terminally ill patients with cancer from a medical center in Taiwan, 460 were recruited and randomly assigned 1:1 to the experimental and control arms. The experimental arm received an interactive ACP intervention tailored to participants' readiness to engage in this process. The control arm received symptom management education. Group allocation was concealed, data collectors were blinded, and treatment fidelity was monitored. Outcome measures included 6 preferred and received LSTs, QoL, anxiety symptoms, and depressive symptoms. Intervention effectiveness was evaluated by intention-to-treat analysis. Results: Participants providing data had died through December 2017. The 2 study arms did not differ significantly in concordance between the 6 preferred and received LSTs examined (odds ratios, 0.966 [95\% Cl, 0.653-1.428] and 1.107 [95\% Cl, 0.690-1.775]). Participants who received the ACP intervention had significantly fewer anxiety symptoms $(\beta,-0.583 ; 95 \% \mathrm{Cl},-0.977$ to $-0.189 ; P=.004)$ and depressive symptoms $(\beta,-0.533 ; 95 \% \mathrm{Cl},-1.036$ to $-0.030 ; P=.038)$ compared with those in the control arm, but QoL did not differ. Conclusions: Our ACP intervention facilitated participants' psychological adjustment to the end-of-life (EoL) care decision-making process, but neither improved QoL nor facilitated EoL care honoring their wishes. The inability of our intervention to improve concordance may have been due to the family power to override patients' wishes in deeply Confucian doctrineinfluenced societies such as Taiwan. Nevertheless, our findings reassure healthcare professionals that such an ACP intervention does not harm but improves the psychological well-being of terminally ill patients with cancer, thereby encouraging physicians to discuss EoL care preferences with patients and involve family caregivers in EoL care decision-making to eventually lead to patient value-concordant EoL cancer care.
\end{abstract}

J Natl Compr Canc Netw 2019;17(4):311-320 doi: 10.6004/jnccn.2018.7106

${ }^{a}$ School of Nursing, Medical College, Chang Gung University; ${ }^{b}$ Department of Nursing, Chang Gung Memorial Hospital at Kaohsiung; 'Division of Hematology-Oncology, Chang Gung Memorial Hospital at Linkou; ${ }^{\mathrm{d} D i v i s i o n}$ of Hematology-Oncology, Chang Gung Memorial Hospital; and ${ }^{\circ}$ Chang Gung University College of Medicine, Tao-Yuan, Taiwan; and fDepartment of International Business, Soochow University, and ${ }^{9}$ College of Nursing, National Taipei University of Nursing and Health Science, Taipei, Taiwan.

\section{Background}

Improving the quality of end-of-life (EoL) care is a priority in healthcare systems. ${ }^{1}$ An essential component of high-quality EoL care is honoring individuals' values and preferences. ${ }^{1,2}$ One strategy to achieve this aim is advance care planning $(\mathrm{ACP})^{3}$ to explore patient preferences for life-sustaining treatments (LSTs) ${ }^{4,5}$ and clarify misunderstandings ${ }^{6}$ about their efficacy at EoL, actively involve patients in EoL care decision-making, and align EoL care with patients' goals and preferences. ${ }^{1,2,4,5}$ Providing such value-based EoL care to terminally ill patients with cancer may not only "individualize" EoL cancer care $^{2}$ and counteract the international trend toward increasingly aggressive and costly EoL care, ${ }^{7-9}$ but also may facilitate adjustment to the uncertainty inherent in EoL care decision-making, thus alleviating anxiety and depressive symptoms and improving quality of life (QoL). ${ }^{10,11}$ The few randomized controlled trials (RCTs) of ACP studies targeting terminally/seriously ill patients ${ }^{12-19}$ have shown that ACP not only increases physicianpatient EoL care discussions, ${ }^{12-19}$ (earlier) completion of advance directives, ${ }^{13,16-18}$ and concordance between preferred and received EoL care, ${ }^{13,16}$ but also does not detrimentally affect patients' anxiety, depression, and QoL. ${ }^{14,18,19}$

However, the robustness and validity of these ACP RCT findings are threatened by several methodological insufficiencies. ${ }^{3,20}$ First, all but one ${ }^{13}$ of the ACP studies we reviewed provided a single-session intervention ${ }^{12,14-19}$ long before the patient's death, ${ }^{12,14,15,18}$ or few $\left(13.67 \%{ }^{16}\right.$ $18.12 \%{ }^{13}$ ) patients died within 6 months after enrollment when concordance between preferred and received EoL care was evaluated. Second, most studies were crosssectional, with only $2^{14,15}$ evaluating intervention outcomes more than once to capture changes as death approached, when EoL care decision-making becomes more relevant. Third, study outcomes commonly centered

See JNCCN.org for supplemental online content. 
on the EoL care process (eg, the content/quality of ACP communication) $)^{12,14,15,18,19}$ and the completion of advance directives, ${ }^{13,16-18}$ but rarely on patient outcomes reflecting high-quality EoL care ${ }^{1,20}$ (eg, EoL care honoring patients' wishes ${ }^{13,15,16,19}$ ), psychological adjustment to the impending death, ${ }^{18,19}$ and better QoL. ${ }^{14}$ Fourth, ACP studies have commonly failed to tailor interventions to participants' readiness to engage in ACP, ${ }^{12,13,16,18}$ directly assess patients' EoL care preferences when evaluating the concordance between preferred and received EoL care, ${ }^{15}$ and explicitly indicate how treatment fidelity was ensured/monitored. ${ }^{12,13,15-19}$

Therefore, this RCT was designed to examine an interactive ACP intervention tailored to participants' readiness to engage in ACP while monitoring/ensuring high treatment fidelity. We hypothesized that our ACP intervention would facilitate concordance between preferred and received LSTs of terminally ill patients with cancer and improve psychologic symptoms and QoL during the dying process compared with an intervention based on symptom management education (control arm).

\section{Patients and Methods}

\section{Design, Settings, and Sample}

Consecutive adult oncology patients were recruited from April 2013 through June 2017 from a medical center in northwest Taiwan when their oncologist first recognized their cancer as terminal (ie, disease continually progressed with distant metastases and was unresponsive to repeated chemotherapy/targeted therapies). Participant oncologists referred patients to research assistants, who were experienced oncology nurses trained by the principal investigator to enroll subjects and collect data. Patients were excluded if they were diagnosed as cognitively incompetent or with psychiatric disorders, were participating in other trials, or were undergoing palliative care, or if their family caregivers were unavailable or declined to participate. Participants were followed through December 2017. This RCT targeted patient-caregiver dyads; only patients' primary outcomes are reported herein. The study protocol (supplemental eAppendix 1, available with this article at JNCCN.org) was approved by the ethics committee of Chang Gung Memorial Hospital, Linkou, Taiwan, and registered at ClinicalTrials.gov (identifier: NCT01912846). All participants provided written informed consent before enrollment.

\section{Sample Size Calculation, Randomization,} and Blinding

Sample size was estimated from observational evidence that patient-physician EoL care discussions (an essential ACP component) increase the likelihood of honoring EoL care preferences of patients with cancer (one of our primary outcomes). ${ }^{11}$ This evidence was used because no ACP RCTs for patients with cancer had reported the same outcomes as ours when the current study was launched. Patients with cancer who reported EoL care discussions with their physicians were more likely to receive EoL care consistent with their preferences (odds ratio [OR], 2.04; $P<.0001) .{ }^{11}$ A sample of 190 dyads per arm would achieve $85 \%$ power to detect a between-arm difference in this primary outcome using 2 -sided tests $(P<.05)$. We did not adjust the alpha values for comparing between-arm multiple primary outcomes and concordances for multiple received/preferred LSTs. Given the $18.5 \%$ attrition in our previous longitudinal study, ${ }^{21} 231$ dyads per arm were needed.

Participants were randomly assigned $1: 1$ to the experimental and control arms using a computergenerated random sequence sealed in consecutively numbered opaque envelopes. To ensure blinding of group assignment, the random allocation sequence was independently generated or assigned by researchers not involved in patient recruitment, interventions, clinical care, and data collection.

\section{Treatments}

This theory-based, individualized, interactive ACP intervention had 5 major components: (1) repeated assessments of participants' readiness to engage in ACP, (2) specific subinterventions tailored to participants' readiness to engage in ACP, (3) facilitation of physicianpatient EoL care discussions, (4) use of a booklet and video educational aid to facilitate understanding of ACP and LSTs at EoL, and (5) psychological support of participants while engaged in EoL care decision-making. During the intervention, participants interacted multiple times with trained interventionists experienced in oncology nursing and palliative care and who were not involved in participants' clinical care. The intervention protocol did not follow a script with prespecified topics. The trained ACP interventionists had the flexibility to provide care tailored to each participant's specific needs at each stage of ACP-engagement readiness and to communicate the patient's concerns and EoL care preferences to clinicians, as needed. Interactions occurred weekly during hospitalization, or monthly at outpatient clinics when participants returned for visits, until they died.

Participants were assessed for ACP-engagement readiness according to the transtheoretical model. ${ }^{22}$ For participants at the precontemplation stage, the individualized subintervention aimed to address their lack of readiness to engage in ACP and to motivate them to consider its relevance. The interventionists explored the values and EoL care preferences of the contemplationstage participant, explained the benefits and burdens of applicable treatments, and encouraged the participants 
to evaluate the benefits and burdens from their own perspective and communicate their preferences and concerns about treatments with their oncologist. For preparation-stage participants, interventionists clarified any unrealistic expectations of continuing/initiating anticancer treatments or LSTs. For participants ready to act on ACP, the interventionists facilitated communication of EoL care preferences to the oncologists to achieve a consensus on EoL care goals and specific treatments that could be used or withheld. For maintenance-stage participants (those who had consolidated their EoL care decisions), interventionists continually provided reassurance regarding the EoL care decisions and made necessary changes in response to health status.

Control-arm participants received a sham treatment of symptom-management education weekly during hospitalization or monthly at outpatient visits until they died. This treatment included (1) symptommanagement education from a trained interventionist experienced in oncology nursing and palliative care, and (2) a booklet and video on symptom management. We hypothesized that despite symptom management being another essential component of high-quality EoL care, ${ }^{1}$ participants' receiving education and a booklet and video on symptom management alone would not impact this study's primary outcomes, ${ }^{23}$ but would parallelize the education received by both study arms as much as possible. Specific booklets and videos were provided to participants in each arm, and interventionists discussed the relevant booklet and video contents with all participants, as needed. Intervention sessions were not audio- or video-recorded for feasibility considerations, but interventionists recorded intervention-treatment components to later evaluate compliance of intervention delivery with the study protocol (supplemental eAppendix 2). This treatment fidelity was monitored throughout the study.

\section{Outcome Measures}

\section{Preferences for LSTs}

Preferences for cardiopulmonary resuscitation, intensive care unit care, chest compression, intubation with mechanical ventilation, nasogastric tube feeding, and intravenous nutrition were assessed using an adapted interview protocol (supplemental eAppendix 3). For each LST, patients were asked whether they (1) wanted, (2) did not want, or (3) were undecided about the treatment.

\section{LSTs Received}

Data on the receipt of the aforementioned 6 LSTs in patients' last month of life were retrieved from medical records and supplemented by caregivers' reports during bereavement follow-ups.

\section{Quality of Life}

Participants' QoL was measured using a modified 13-item McGill QoL Questionnaire (MQoL). ${ }^{24}$ The original MQoL stresses psychologic, social, and existential well-being. We omitted 3 items (the 3 most distressing symptoms) to avoid overlap with the potential effect of symptom distress on participants' QoL. Total scores for this modified MQoL ranged from 0 to 130; higher scores indicated a better QoL.

\section{Anxiety and Depressive Symptoms}

Anxiety and depressive symptoms were measured by the Hospital Anxiety and Depression Scale (HADS). ${ }^{25}$ The 14-item HADS, the most widely used tool for assessing psychological distress in patients with cancer undergoing palliative care ${ }^{26}$ has 7 items measuring anxiety (HADS-A) and 7 measuring depression (HADS-D). HADS assesses psychological symptoms rather than physiological symptoms, thus avoiding confounding measures that may overestimate anxiety or depression severity for patients with cancer who commonly experience multiple physical symptoms. The HADS-A and HADS-D subscales each have a total score ranging from 0 to 21 ; higher scores indicate more anxiety or depressive symptoms.

\section{Covariate Measures}

To evaluate the effectiveness of our ACP intervention, we adjusted for several covariates shown to influence QoL, anxiety symptoms, and depressive symptoms: treatment dose (ie, number of intervention treatments provided), proximity to death ${ }^{27,28}$ (ie, the period between death and assessment [ie, 1-30, 31-90, 91-180, 181-365, 366-545, and $\geq 546$ days before death], which are conventional periods for estimating survival), ${ }^{29}$ known time-varying covariates that may vary in each assessment (ie, physical symptom distress ${ }^{30}$ and functional dependence), ${ }^{31}$ and time-invariant variables (ie, comorbidity, age, and sex; see supplemental eAppendix 4).

\section{Data Collection}

Participants' characteristics were assessed at baseline (before random assignment). Data on outcome measures (LST preferences, QoL, anxiety symptoms, and depressive symptoms) and time-varying covariates were collected at baseline and every 3 to 4 weeks afterward until participants withdrew or died. Data collectors were blinded to participants' group assignment.

\section{Statistical Analysis}

Between-arm homogeneity at baseline was examined by chi-square and independent $t$ tests. LST preferences were dichotomized into "want" / do not want" treatment, with those indicating "undecided" counted as wanting that treatment because the clinical default is to provide LSTs 
unless specifically refused. ${ }^{32}$ Concordance was evaluated by cross-tabulating each LST last reported before death and LST received in participants' last month of life. Concordance was expressed as both a percentage (received/preferred LSTs) and a kappa coefficient for chance-corrected agreement. ${ }^{33}$ Kappa values were categorized as poor $(\leq 0.20)$, fair $(0.21-0.40)$, moderate (0.41-0.60), substantial (0.61-0.80), or almost perfect (0.81-1.00). ${ }^{34}$ Between-arm differences in concordance between preferred and received LSTs were expressed by an OR with $95 \%$ CI.

Effects of the ACP intervention on the 3 continuous outcome variables (QoL, anxiety symptoms, depressive symptoms) during the dying process were simultaneously evaluated by intention-to-treat analysis ${ }^{35}$ using multivariate hierarchical linear modeling (MHLM ${ }^{36}$ by HLM 7.0 to control for the inflation of type I errors from multiple outcome comparisons. Furthermore, MHLM allows different waves of data across patients to accommodate variable numbers of follow-up points, inconsistent time intervals between subsequent data collections, and missing data for the dependent variables, eliminating the need to delete observations in analyses. ${ }^{36}$ MHLM uses random intercepts to account for within-subject correlations of repeated observations from each patient. ${ }^{36}$ The MHLM in this study controlled for treatment dose, proximity to death, and time-invariant and lagged time-varying covariates (functional dependence, physical symptom distress, anxiety symptoms, depressive symptoms, and QoL measured in the previous wave of assessment) to ensure a clear time sequence of effects on the 3 outcomes.

\section{Results}

\section{Patient Characteristics}

Of 795 patients approached, 460 (57.86\%) were enrolled and randomly assigned 1:1 to the experimental and control arms (Figure 1). Nonenrollment was primarily due to family caregivers' unavailability and patients' weakness. The final sample included 215 participants per arm who died by the end of the study. Between-arm baseline characteristics, LST preferences, QoL, anxiety symptoms, and depressive symptoms were balanced at randomization (supplemental eAppendix 5) and in the final sample (Table 1). Experimental and control arm participants received on average 5.99 ACP intervention sessions (SD, 6.11) and 5.43 sham treatment sessions (SD, 6.45), respectively. After enrollment, experimental and control arm participants survived 147.33 (SD, 155.01 ) and 127.39 days (SD, 151.66) and were assessed 5.19 (SD, 4.75) and 4.93 times (SD, 5.35), respectively. The last assessment was on average 27.69 days (SD, 52.99) before death.

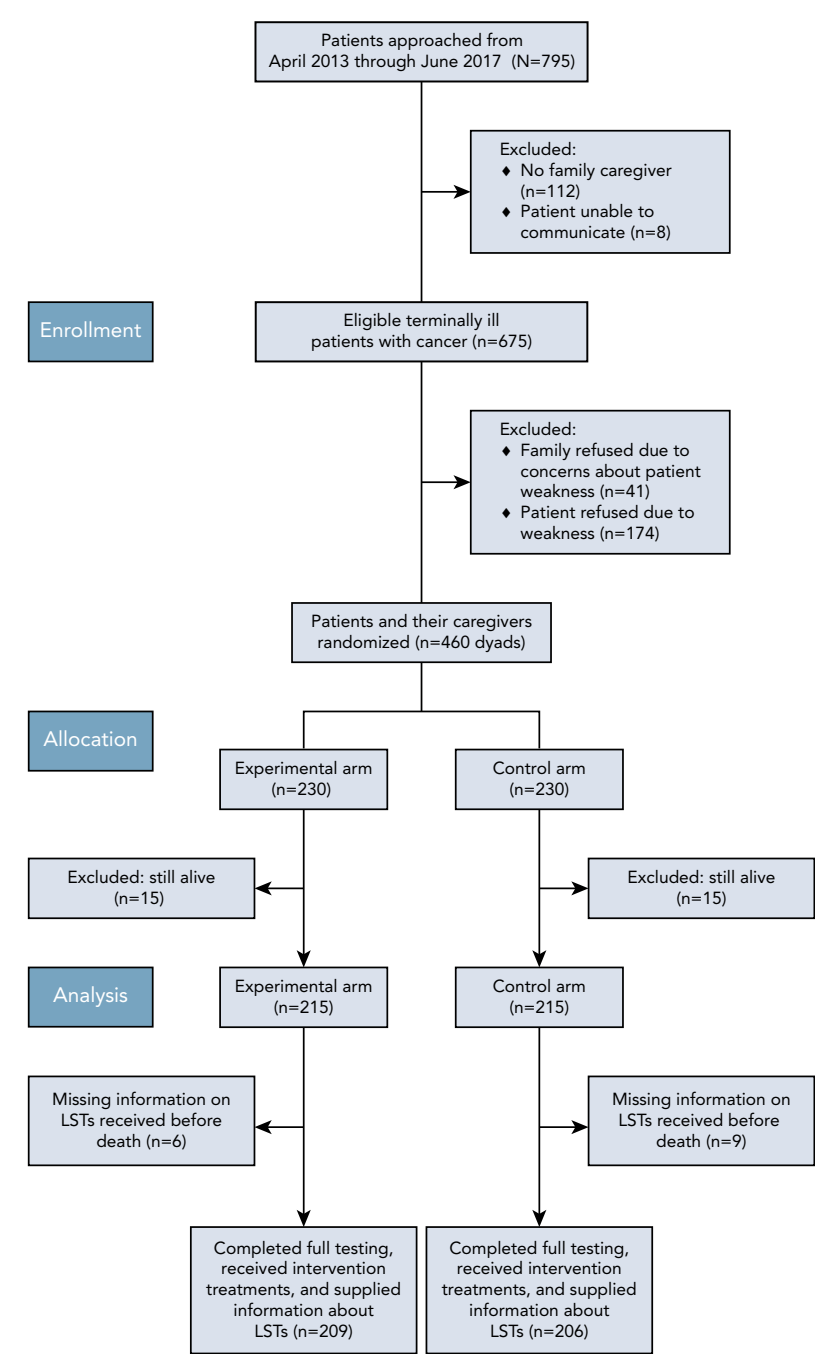

Figure 1. CONSORT diagram.

Abbreviation: LSTs, life-sustaining treatments.

\section{Preferred and Received LSTs}

The ACP intervention did not facilitate concordance between preferred and received LSTs. Concordance between participants' last-assessed LST preferences and LSTs received in the last month was $58.2 \%$ to $77.5 \%$ and $59.0 \%$ to $75.7 \%$ for the experimental and control arms, respectively (Table 2 ), with patients receiving fewer LSTs than they preferred (data not shown). Concordance between preferred and received LSTs was poor to fair for

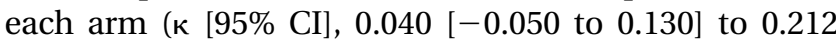
[0.098-0.326], and 0.019 [-0.063 to 0.101$]$ to 0.224 [0.110-0.338] for the experimental and the control arms, respectively. Between-arm concordance did not differ significantly for any of the 6 preferred and received LSTs examined (OR [95\% CI], $0.966[0.653-1.428]$ to 1.107 [0.690-1.775]). 


\section{Table 1. Patient Characteristics $(\mathrm{N}=430)$}

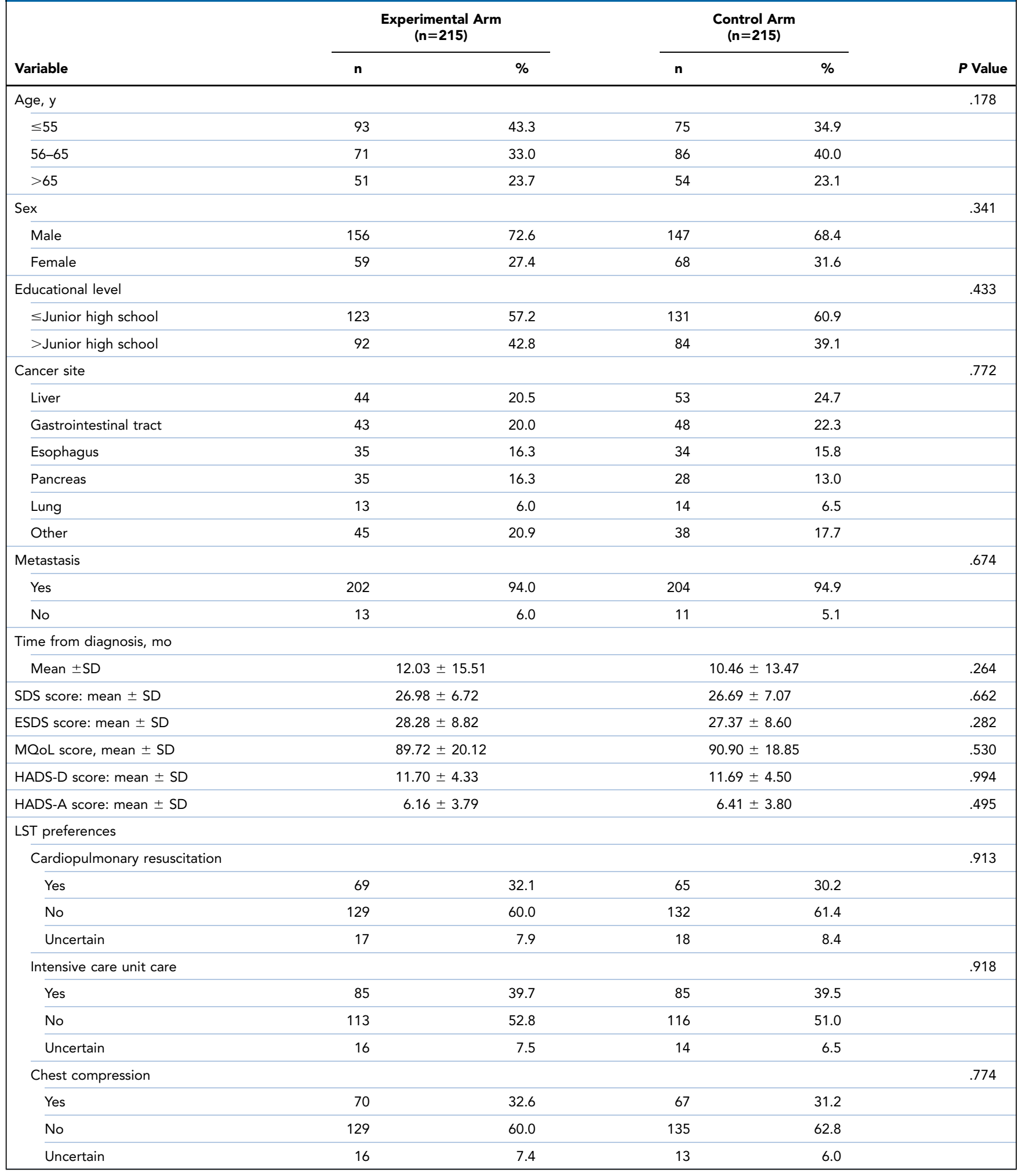
Scale-Depression; LST, life-sustaining treatment; MQoL, McGill Quality of Life Questionnaire; SDS, Symptom Distress Scale. 


\section{Table 1. Patient Characteristics ( $N=430)$ (cont.)}

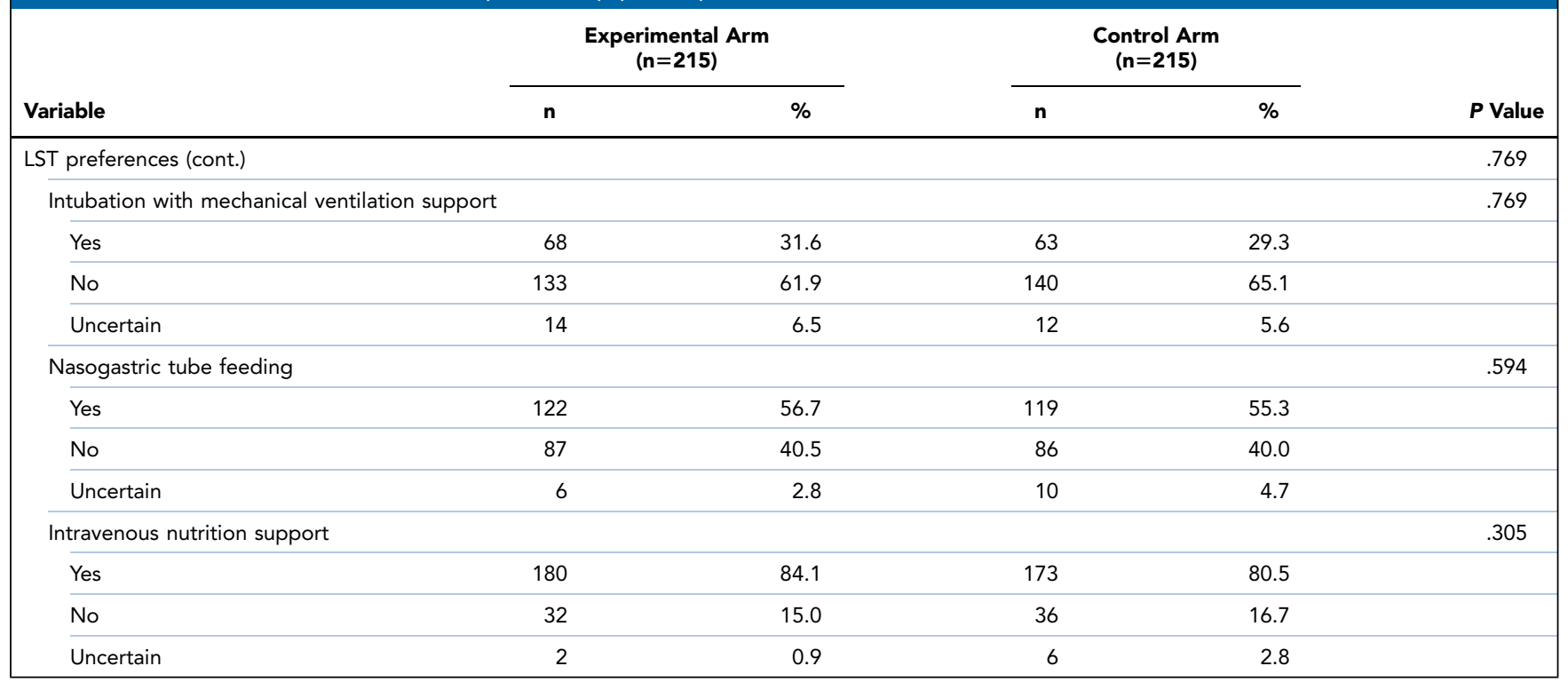

Abbreviations: ESDS, Enforced Social Dependency Scale; HADS-A, Hospital Anxiety and Depression Scale-Anxiety; HADS-D, Hospital Anxiewty and Depression Scale-Depression; LST, life-sustaining treatment; MQoL, McGill Quality of Life Questionnaire; SDS, Symptom Distress Scale.

\section{Anxiety Symptoms, Depressive Symptoms,} and QoL

ACP intervention improved anxiety symptoms and depressive symptoms but not QoL that patients preferred regarding their dying process. Unadjusted MQoL, HADS-A, and HADS-D scores for both arms are shown in Figure 2. After controlling for identified covariates, the simultaneous MHLM analysis of the 3 outcomes showed no significant difference in QoL between the experimental and the control arms (Table 3). However, the experimental arm participants reported significantly fewer anxiety symptoms ( $\beta$ [95\% CI], $-0.583[-0.977$ to 0.189 ]; $P=.004$ ) and depressive symptoms ( $\beta$ [95\% CI], 0.533 [ -1.036 to -0.030$] ; P=.038$ ) than the control arm participants.

\section{Discussion}

The results support our hypothesis that an individualized, interactive ACP intervention would lighten anxiety and depressive symptoms in terminally ill patients with cancer who are facing EoL and making EoL care decisions. However, the ACP intervention did not facilitate concordance between patients' preferred and received LSTs, nor did it improve their QoL at EoL. The inability of the ACP intervention to honor participants' LST preferences is consistent with reports on patients with life-limiting noncancer disease ${ }^{15}$ and on overall patientassessed goal-concordant care, ${ }^{19}$ but not with the significant positive impact reported for elderly patients ${ }^{13}$ or nursing home residents. ${ }^{16}$ Our finding of poor-to-fair concordance between patients' preferred and received

Table 2. Effectiveness of ACP Intervention in Facilitating Concordance With LSTs

\begin{tabular}{|c|c|c|c|c|c|c|c|c|}
\hline \multirow[b]{3}{*}{ LST } & \multicolumn{8}{|c|}{ Concordance Between Preferred and Received LSTs } \\
\hline & \multicolumn{3}{|c|}{ Experimental Arm } & \multicolumn{3}{|c|}{ Control Arm } & \multicolumn{2}{|c|}{ Arm Difference } \\
\hline & $\mathbf{n}$ & $\%$ & $\kappa(95 \% \mathrm{Cl})$ & $\mathbf{n}$ & $\%$ & к $(95 \% \mathrm{Cl})$ & OR $(95 \% \mathrm{Cl})$ & $P$ Value \\
\hline Cardiopulmonary resuscitation & 208 & 69.7 & $0.077(-0.023$ to 0.177$)$ & 205 & 68.3 & $0.061(-0.029$ to 0.151$)$ & $1.069(0.704-1.622)$ & .755 \\
\hline Intensive care unit care & 207 & 62.3 & $0.054(-0.044$ to 0.152$)$ & 205 & 61.0 & $0.030(-0.060$ to 0.120$)$ & $1.058(0.711-1.575)$ & .779 \\
\hline Chest compression & 207 & 69.6 & $0.040(-0.050$ to 0.130$)$ & 204 & 69.1 & $0.019(-0.063$ to 0.101$)$ & $1.021(0.671-1.553)$ & .922 \\
\hline Intubation with mechanical ventilation & 207 & 69.6 & $0.080(-0.045$ to 0.205$)$ & 205 & 69.6 & $0.051(-0.059$ to 0.161$)$ & $0.991(0.651-1.508)$ & .966 \\
\hline Nasogastric tube feeding & 208 & 58.2 & $0.212(0.098-0.326)$ & 205 & 59.0 & $0.224(0.110-0.338)$ & $0.966(0.653-1.428)$ & .861 \\
\hline Intravenous nutrition support & 200 & 77.5 & $0.174(0.009-0.339)$ & 185 & 75.7 & $0.095(-0.066$ to 0.256$)$ & $1.107(0.690-1.775)$ & .673 \\
\hline
\end{tabular}

Abbreviations: ACP, advance care planning; LSTs, life-sustaining treatments; OR, odds ratio. 


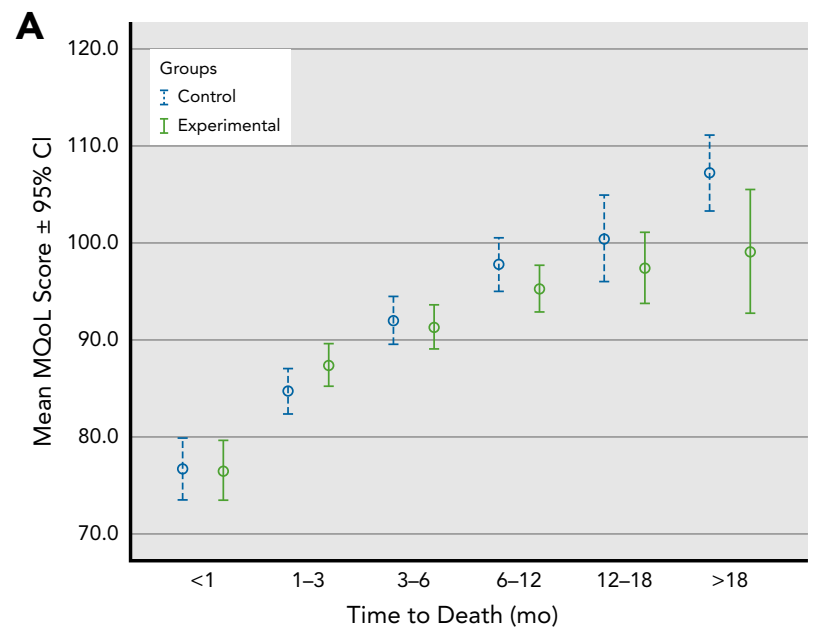

B

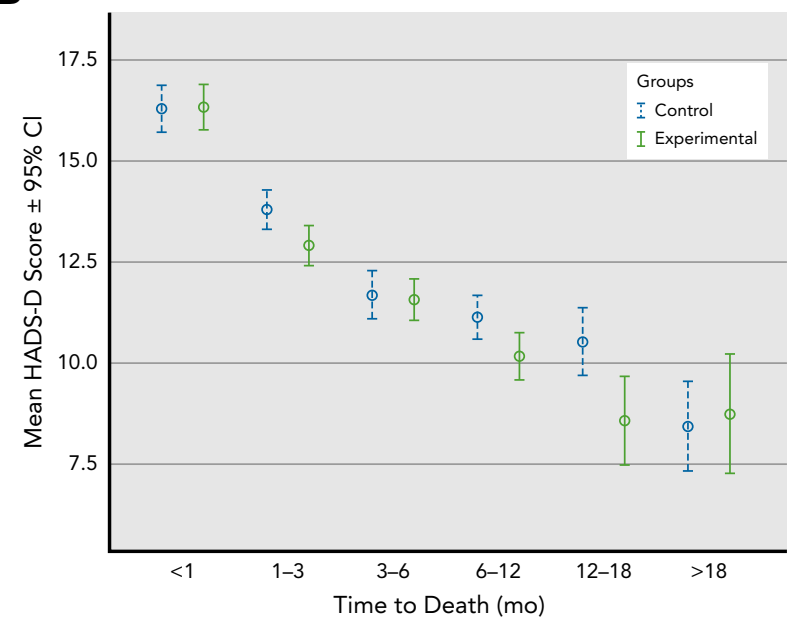

C

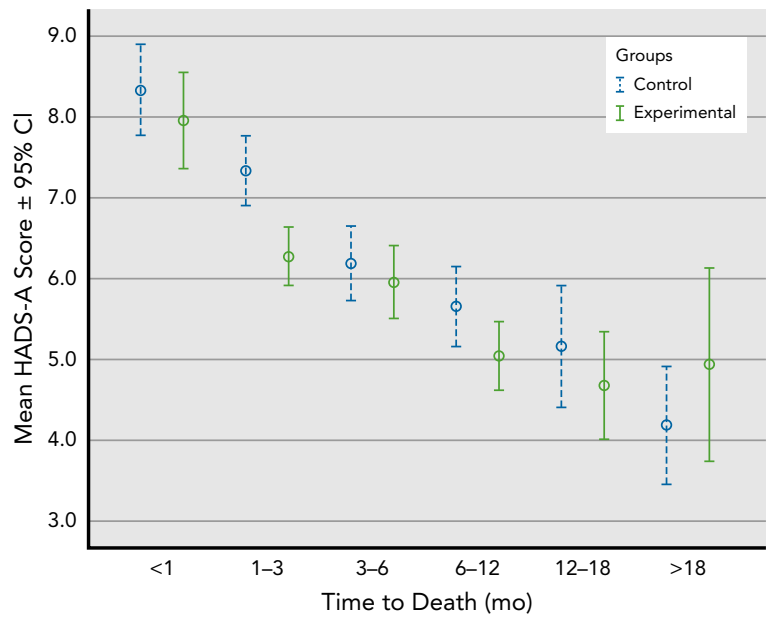

Figure 2. Unadjusted mean $(95 \% \mathrm{Cl})$ scores for (A) quality of life, (B) depressive symptoms, and (C) anxiety symptoms during the dying trajectory.

Abbreviations: HADS-A, Hospital Anxiety and Depression Scale-Anxiety; HADS-D, Hospital Anxiety and Depression Scale-Depression; MQoL, McGill Quality of Life Questionnaire. ${ }^{24}$
LSTs may be attributable to the family power to override the autonomy/wishes of conscious, competent patients. ${ }^{37}$ This power is commonly exercised in medical care decision-making, including EoL care, ${ }^{38}$ in deeply Confucian doctrine-influenced societies such as Taiwan. In future studies, we will examine how our ACP intervention influenced family caregivers' attitudes toward honoring and supporting their relative's LST preferences. In addition, we repeatedly assessed participants' LST preferences regarding their dying process because the extent to which their EoL care preferences are honored may be distorted if preferences are measured long before death, a common approach of existing research. ${ }^{11,13,15}$ However, this repeated assessment (testing) ${ }^{39}$ may have threatened the internal validity of our study by sensitizing and motivating control-arm participants to prepare for their EoL care, thereby neutralizing/minimizing the intervention's effectiveness in facilitating concordance between patients' preferred and received LSTs.

The ACP intervention significantly facilitated patients' psychological adjustment while they were deciding about their EoL care. Our results demonstrate that the ACP intervention not only caused no psychological harm, as previously reported, ${ }^{18,19}$ but that it also reduced anxiety and depressive symptoms for those in the experimental arm compared with the control arm. This success is probably attributable to the strategies of actively exploring participants' EoL care values, goals, and preferences; tailoring the ACP intervention to their readiness to engage in ACP; and sensitively providing psychological support for those struggling with making such emotion-laden decisions. Worldwide, especially in Asian countries, well-intentioned physicians tend to omit or delay EoL care discussions with patients to avoid disturbing or overwhelming them, ${ }^{40}$ leading to considerable discrepancies between patients' preferred and actual involvement in EoL care discussions, ${ }^{41}$ especially for highly anxious or depressed patients. ${ }^{41}$ Avoiding EoL care discussions may in turn predispose patients to experience further anxiety or depressive symptoms. Our intervention may have ended this vicious cycle by facilitating psychological adjustment while patients were making EoL care decisions. However, the intervention did not improve patients' QoL throughout the dying process. Improving QoL at EoL may require components not included this ACP intervention, such as adequate and effective symptom management ${ }^{42}$ or comprehensive and early integration of palliative care into standard oncology care. $^{43}$

Strengths of our study include its interactive intervention tailored to participants' readiness to engage in $\mathrm{ACP},{ }^{4}$ intervening throughout the dying process, ${ }^{20}$ evaluating high-quality EoL care outcomes based on most participants' self-reports, and ensuring internal validity 
Table 3. Impact of ACP Intervention on QoL, Anxiety Symptoms, and Depressive Symptoms

\begin{tabular}{|c|c|c|c|c|c|c|c|c|c|c|c|c|}
\hline \multirow[b]{2}{*}{ Variable } & \multicolumn{4}{|c|}{ QoL } & \multicolumn{4}{|c|}{ Anxiety Symptoms } & \multicolumn{4}{|c|}{ Depressive Symptoms } \\
\hline & $\beta$ & \multicolumn{2}{|c|}{$95 \% \mathrm{Cl}$} & $P$ Value & $\boldsymbol{\beta}$ & \multicolumn{2}{|c|}{$95 \% \mathrm{Cl}$} & $P$ Value & $\boldsymbol{\beta}$ & \multicolumn{2}{|c|}{$95 \% \mathrm{Cl}$} & $P$ Value \\
\hline \multicolumn{13}{|l|}{ Arm } \\
\hline Experimental & 1.689 & -0.578 & 3.956 & .144 & -0.583 & -0.977 & -0.189 & .004 & -0.533 & -1.036 & -0.030 & .038 \\
\hline Control & Ref & & & & Ref & & & & Ref & & & \\
\hline \multicolumn{13}{|l|}{ Proximity to death, $d$} \\
\hline$\leq 30$ & Ref & & & & Ref & & & & Ref & & & \\
\hline $31-90$ & 7.527 & 5.575 & 9.478 & $<.001$ & -1.082 & -1.499 & -0.666 & $<.001$ & -2.202 & -2.678 & -1.727 & $<.001$ \\
\hline $91-180$ & 12.143 & 9.999 & 14.286 & $<.001$ & -1.672 & -2.121 & -1.223 & $<.001$ & -3.682 & -4.200 & -3.164 & $<.001$ \\
\hline $181-365$ & 15.548 & 13.079 & 18.017 & $<.001$ & -2.219 & -2.722 & -1.717 & $<.001$ & -4.411 & -5.000 & -3.822 & $<.001$ \\
\hline $366-545$ & 18.906 & 15.443 & 22.369 & $<.001$ & -2.957 & -3.657 & -2.256 & $<.001$ & -5.748 & -6.573 & -4.924 & $<.001$ \\
\hline$\geq 546$ & 18.976 & 14.518 & 23.435 & $<.001$ & -2.778 & -3.675 & -1.881 & $<.001$ & -5.679 & -6.738 & -4.620 & $<.001$ \\
\hline \multicolumn{13}{|l|}{ Lagged time-varying variables } \\
\hline Symptom distress ${ }^{\mathrm{a}}$ & -0.030 & -0.163 & 0.103 & .657 & 0.017 & -0.011 & 0.045 & .230 & 0.030 & -0.003 & 0.062 & .070 \\
\hline Functional dependence ${ }^{b}$ & 0.047 & -0.072 & 0.166 & .438 & 0.017 & -0.007 & 0.041 & .169 & 0.026 & -0.003 & 0.054 & .077 \\
\hline $\mathrm{QoL}^{\mathrm{c}}$ & 0.383 & 0.314 & 0.452 & $<.001$ & -0.036 & -0.050 & -0.022 & $<.001$ & -0.003 & -0.019 & 0.014 & .748 \\
\hline Depressive symptoms ${ }^{d}$ & 0.245 & -0.031 & 0.522 & .082 & -0.026 & -0.084 & 0.031 & .366 & 0.281 & 0.214 & 0.347 & $<.001$ \\
\hline Anxiety symptoms ${ }^{e}$ & -0.857 & -1.173 & -0.541 & $<.001$ & 0.289 & 0.223 & 0.355 & $<.001$ & 0.078 & 0.002 & 0.155 & .045 \\
\hline Treatment dose & -0.126 & -0.280 & 0.028 & .109 & 0.008 & -0.022 & 0.038 & .605 & 0.012 & -0.024 & 0.048 & .525 \\
\hline \multicolumn{13}{|l|}{ Sex } \\
\hline Male & -0.252 & -2.717 & 2.213 & .842 & -0.531 & -0.957 & -0.105 & .015 & -0.080 & -0.626 & 0.465 & .773 \\
\hline Female & Ref & & & & Ref & & & & Ref & & & \\
\hline \multicolumn{13}{|c|}{ Age, y (dichotomized at median age) } \\
\hline$>57$ & 6.254 & 3.869 & 8.639 & $<.001$ & -0.387 & -0.805 & 0.030 & .068 & 0.157 & -0.374 & 0.688 & .562 \\
\hline$\leq 57$ & Ref & & & & Ref & & & & Ref & & & \\
\hline \multicolumn{13}{|l|}{ Comorbidities ${ }^{f}$} \\
\hline 0 & Ref & & & & Ref & & & & Ref & & & \\
\hline 1 & 0.997 & -1.795 & 3.790 & .484 & -0.028 & -0.511 & 0.454 & .909 & -0.677 & -1.295 & -0.059 & .032 \\
\hline 2 & 0.367 & -2.741 & 3.475 & .817 & 0.229 & -0.307 & 0.766 & .402 & -0.206 & -0.894 & 0.481 & .566 \\
\hline$\geq 3$ & 2.614 & -1.329 & 6.557 & .194 & 0.088 & -0.609 & 0.785 & .805 & -0.184 & -1.065 & 0.697 & .682 \\
\hline
\end{tabular}

Abbreviations: ACP, advance care planning; QoL, quality of life.

aMeasured with Symptom Distress Scale.

${ }^{b}$ Measured with Enforced Social Dependency Scale.

cMeasured with McGill QoL Questionnaire.

dMeasured with Hospital Anxiety and Depression Scale-Depression.

eMeasured with Hospital Anxiety and Depression Scale-Anxiety.

${ }^{f}$ Charlson-Deyo comorbidity index.

through using a rigorous design and monitoring treatment fidelity. However, this RCT was conducted at a Taiwanese hospital using a sample of terminally ill patients with cancer who had a family caregiver and who were referred to this study by their oncologist, limiting the generalization of these findings to national and international target populations without an available family caregiver. We focused on the patient participants and did not consider family caregivers' perspectives. Future studies should evaluate how ACP interventions influence family caregivers' understanding, support, and endorsement of patients' LST preferences to achieve patient value-concordant EoL care. ${ }^{44}$ Furthermore, terminally ill patients' indecision when making EoL care choices indicates their ambivalence about LSTs. Categorizing "undecided" responses as treatment preferences may have inflated the preference rate (approximately 10\%; see Table 1), but removing them from analyses may not bring undecided patients' preferences to the attention of clinicians, who would miss the opportunity to meet the needs of these patients at EoL. Further research should explore the role of uncertainty 
in facilitating or hindering concordance between the preferred and received EoL care of terminally ill patients with cancer and evaluate interventions to eliminate their uncertainty in making EoL care decisions. ${ }^{45}$

\section{Conclusions}

This interactive intervention tailored to the readiness of terminally ill patients with cancer to engage in ACP during the dying process facilitated their psychological adjustment to EoL care decision-making despite not influencing concordance between their preferred and received LSTs and not improving their QoL before death. These findings assure healthcare professionals that such an ACP intervention does not harm the psychological well-being of terminally ill patients with cancer but instead improves it. With this assurance, clinicians can systematically adopt and integrate ACP interventions into clinical care ${ }^{40}$ to respect patients' desire for active participation in EoL care discussions ${ }^{41}$ and to facilitate physician-patient EoL care discussions early in the patient's disease trajectory. ${ }^{1}$ We suggest involving family caregivers in EoL care decision-making and considering their LST preferences, ${ }^{44}$ which may eventually lead to EoL cancer care that is concordant with patient values.

\section{Submitted July 16, 2018; accepted for publication November 9, 2018.}

Author contributions: Study concept and design: All authors. Provision of study material or patients: J.S. Chen, Chou, Chang, Hsieh. Data acquisition: Al authors. Data analysis and interpretation: Tang, Wen. Manuscript preparation: Tang. Final approval: All authors.

Disclosures: The authors have not received any financial consideration from any person or organization to support the preparation, analysis, results, or discussion of this article.

Funding: This work was supported by the National Health Research Institutes (NHRI-EX106-10208PI), Ministry of Science and Technology (MOST 105-2314B-182-040-MY3), and Chang Gung Memorial Hospital (BMRP888).

Correspondence: Siew Tzuh Tang, DNSc, School of Nursing, Medical College, Chang Gung University, 259 Wen-Hwa 1st Road, Kwei-Shan, Tao-Yuan, Taiwan, 333, R.O.C. Email: sttang@mail.cgu.edu.tw

\section{References}

1. Institute of Medicine. Dying in America: Improving Quality and Honoring Individual Preferences Near the End of Life. Washington, DC:The National Academies Press; 2014

2. Peppercorn JM, Smith TJ, Helft PR, et al. Toward individualized care for patients with advanced cancer. J Clin Oncol 2011;29:755-760.

3. Johnson $S$, Butow $P$, Kerridge I, et al. Advance care planning for cancer patients: a systematic review of perceptions and experiences of patients, families, and healthcare providers. Psychooncology 2016;25:362-386.

4. Rietjens JA, Sudore RL, Connolly M, et al. Definition and recommendations for advance care planning: an international consensus supported by the European Association for Palliative Care. Lancet Oncol 2017: 18:e543-551.

5. Sudore RL, Heyland DK, Lum HD, et al. Outcomes that define successful advance care planning: a Delphi panel consensus. J Pain Symptom Manage 2018;55:245-255.

6. Heyland DK, Frank C, Groll D, et al. Understanding cardiopulmonary resuscitation decision making: perspectives of seriously ill hospitalized patients and family members. Chest 2006;130:419-428.

7. Bekelman JE, Halpern SD, Blankart CR, et al. Comparison of site of death, health care utilization, and hospital expenditures for patients dying with cancer in 7 developed countries. JAMA 2016;315:272-283.

8. Falchook AD, Dusetzina $S B$, Tian F, et al. Aggressive end-of-life care for metastatic cancer patients younger than age 65 years. J Natl Cancer Inst 2017;109:djx028.

9. Hung YN, Liu TW, Wen FH, et al. Escalating healthcare expenditures in cancer decedents' last year of life: a decade of evidence from a retrospective population-based cohort study in Taiwan. Oncologist 2017; 22:460-469.

10. Wright AA, Zhang B, Ray A, et al. Associations between end-of-life discussions, patient mental health, medical care near death, and caregiver bereavement adjustment. JAMA 2008;300:1665-1673.

11. Mack JW, Weeks JC, Wright AA, et al. End-of-life discussions, goal attainment, and distress at the end of life: predictors and outcomes of receipt of care consistent with preferences. J Clin Oncol 2010; 28:1203-1208.

12. Au DH, Udris EM, Engelberg RA, et al. A randomized trial to improve communication about end-of-life care among patients with COPD. Chest 2012;141:726-735.

13. Detering $K M$, Hancock $A D$, Reade $M C$, et al. The impact of advance care planning on end of life care in elderly patients: randomised controlled trial. BMJ 2010;340:c1345.

14. Epstein RM, Duberstein PR, Fenton JJ, et al. Effect of a patient-centered communication intervention on oncologist-patient communication, quality of life, and health care utilization in advanced cancer: the VOICE randomized clinical trial. JAMA Oncol 2017;3:92-100.

15. Kirchhoff KT, Hammes BJ, Kehl KA, et al. Effect of a disease-specific planning intervention on surrogate understanding of patient goals for future medical treatment. J Am Geriatr Soc 2010;58:1233-1240.

16. Morrison RS, Chichin E, Carter J, et al. The effect of a social work intervention to enhance advance care planning documentation in the nursing home. J Am Geriatr Soc 2005;53:290-294.

17. Pearlman RA, Starks $\mathrm{H}$, Cain KC, et al. Improvements in advance care planning in the Veterans Affairs System: results of a multifaceted intervention. Arch Intern Med 2005:165:667-674.

18. Stein RA, Sharpe L, Bell ML, et al. Randomized controlled trial of structured intervention to facilitate end-of-life decision making in patients with advanced cancer. J Clin Oncol 2013;31:3403-3410.

19. Curtis JR, Downey L, Back AL, et al. Effect of a patient and clinician communication-priming intervention on patient-reported goals-of-care discussions between patients with serious illness and clinicians: a randomized clinical trial. JAMA Intern Med 2018;178:930-940.

20. Cherny N. Palliative care: lemonade from lemons: exploring the results of the VOICE study. Nat Rev Clin Oncol 2017;14:8-10.

21. Tang ST, Wen FH, Chang WC, et al. Preferences for life-sustaining treatments examined by hidden Markov modeling are mostly stable in terminally ill cancer patients' last 6 months of life. J Pain Symptom Manage 2017;54:628-636.

22. Prochaska JO, Velicer WF. The transtheoretical model of health behavior change. Am J Health Promot 1997;12:38-48.

23. Tamayo-Velázquez MI, Simón-Lorda P, Villegas-Portero R, et al. Interventions to promote the use of advance directives: an overview of systematic reviews. Patient Educ Couns 2010;80:10-20.

24. Cohen SR, Mount BM, Tomas JJ, et al. Existential well-being is an important determinant of quality of life. Evidence from the McGill Quality of Life Questionnaire. Cancer 1996;77:576-586.

25. Zigmond AS, Snaith RP. The hospital anxiety and depression scale. Acta Psychiatr Scand 1983;67:361-370.

26. Wasteson E, Brenne E, Higginson IJ, et al. Depression assessment and classification in palliative cancer patients: a systematic literature review. Palliat Med 2009;23:739-753.

27. Elmqvist MA, Jordhøy MS, Bjordal $K$, et al. Health-related quality of life during the last three months of life in patients with advanced cancer. Support Care Cancer 2009;17:191-198.

28. Lo C, Zimmermann C, Rydall A, et al. Longitudinal study of depressive symptoms in patients with metastatic gastrointestinal and lung cancer. J Clin Oncol 2010;28:3084-3089. 
29. Christakis NA, Escarce JJ. Survival of Medicare patients after enrollment in hospice programs. N Engl J Med 1996;335:172-178.

30. Astrup GL, Hofso K, Bjordal K, et al. Patient factors and quality of life outcomes differ among four subgroups of oncology patients based on symptom occurrence. Acta Oncol 2017;56:462-470.

31. Seow H, Barbera L, Sutradhar R, et al. Trajectory of performance status and symptom scores for patients with cancer during the last six months of life. J Clin Oncol 2011;29:1151-1158.

32. Rose $\mathrm{JH}, \mathrm{O}^{\prime}$ Toole EE, Dawson NV et al. Perspectives, preferences, care practices, and outcomes among older and middle-aged patients with late-stage cancer. J Clin Oncol 2004;22:4907-4917.

33. Bland JM, Altman DG. Statistical methods for assessing agreement between two methods of clinical measurement. Lancet 1986;327:307-310.

34. Landis JR, Koch GG. The measurement of observer agreement for categorical data. Biometrics 1977;33:159-174.

35. Hollis S, Campbell F. What is meant by intention to treat analysis? Survey of published randomised controlled trials. BMJ 1999;319:670-674.

36. Tate RL, Pituch KA. Multivariate hierarchical linear modeling in randomized field experiments. J Experimental Educ 2007;75: 317-337.

37. Liu TW, Wen FH, Wang $\mathrm{CH}$, et al. Terminally ill Taiwanese cancer patients' and family caregivers' agreement on patterns of life-sustaining treatment preferences is poor to fair and declines over a decade: results from two independent cross-sectional studies. J Pain Symptom Manage 2017;54: 35-45

38. Yang $Y$. A family-oriented Confucian approach to advance directives in end-of-life decision making for incompetent elderly patients. In: Fan RP ed. Family-Oriented Informed Consent: East Asian and American Perspectives. Basel, Switzerland: Springer International Publishing; 2015:257-270.

39. Campbell DT. Factors relevant to the validity of experiments in social settings. Psychol Bull 1957;54:297-312.

40. Bernacki RE, Block SD. Communication about serious illness care goals: a review and synthesis of best practices. JAMA Intern Med 2014; 174:1994-2003.

41. Fakhri S, Engelberg RA, Downey $L$, et al. Factors affecting patients' preferences for and actual discussions about end-of-life care. J Pain Symptom Manage 2016;52:386-394.

42. Basch E, Deal AM, Kris MG, et al. Symptom monitoring with patientreported outcomes during routine cancer treatment: a randomized controlled trial. J Clin Oncol 2016;34:557-565.

43. Haun MW, Estel S, Rücker G, et al. Early palliative care for adults with advanced cancer. Cochrane Database Syst Rev 2017;12:CD011129.

44. Fried $T$, Zenoni $M$, lannone $L$. A dyadic perspective on engagement in advance care planning. J Am Geriatr Soc 2017;65:172-178.

45. Barnato AE. Challenges in understanding and respecting patients' preferences. Health Aff (Millwood) 2017;36:1252-1257.

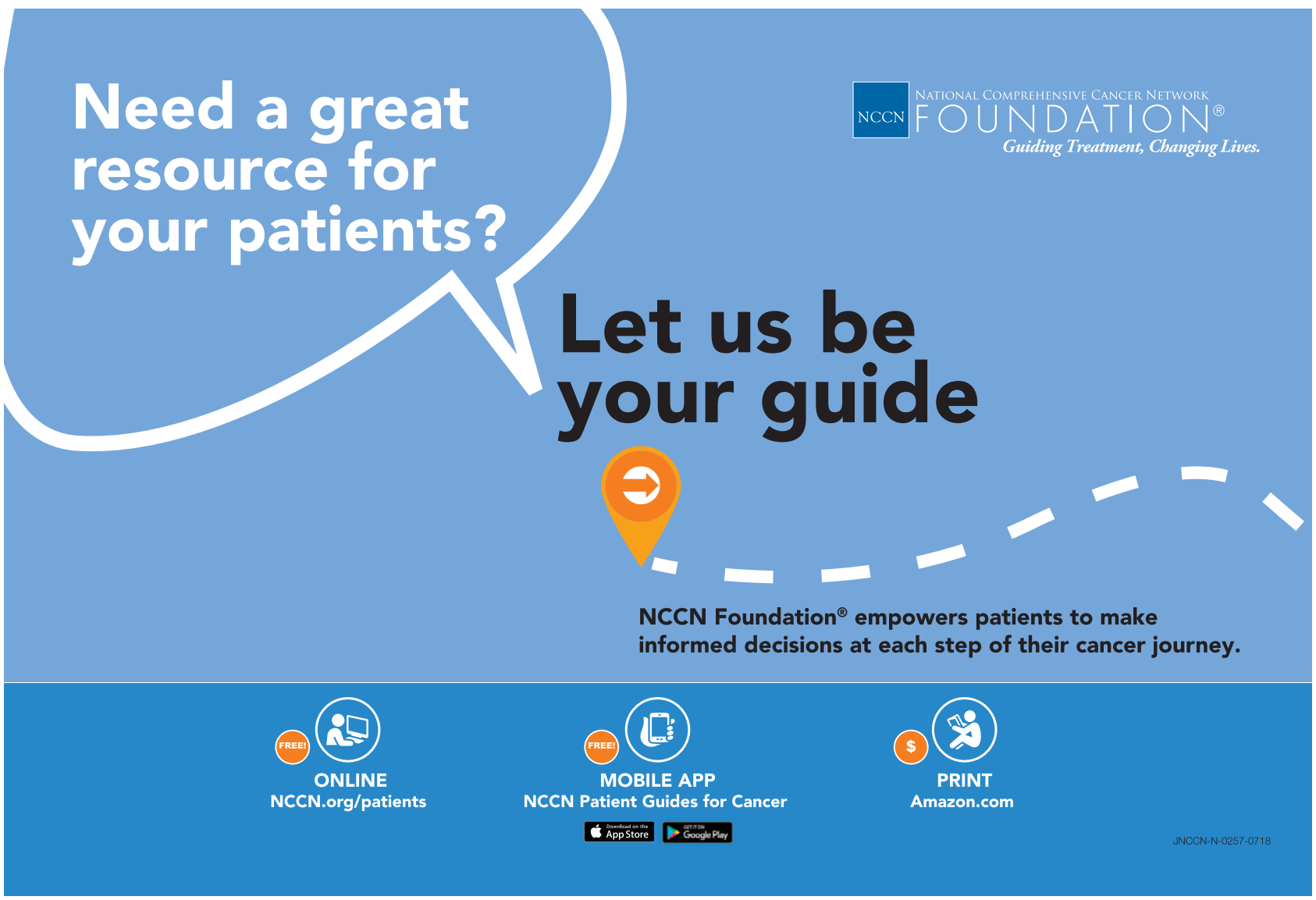

